\title{
Eficacia y reproducibilidad de la técnica de mapeo linfático con biopsia de ganglio centinela en melanoma maligno
}

\author{
Efficacy and reproducibility of lymphatics mapping technique with sentinel lymph node \\ biopsy in melanoma
}

\author{
Manuel I. España*, Franco Signorini, Marcos Marani, Alvaro Alcaraz, Santiago Reimondez, \\ $M^{a}$ Eugenia Romero y Lucio Obeide \\ Hospital Privado Universitario de Córdoba, Córdoba, Argentina
}

\section{Resumen}

Introducción: El estudio del ganglio centinela (GC) en el melanoma maligno es un procedimiento que busca la identificación del primer ganglio al cual drena el sector cutáneo comprometido a fin de evitar linfadenectomías innecesarias. El presente estudio documenta la frecuencia de identificación del GC y la relación entre GC positivo (GCP) y recurrencia, entre el índice de Breslow (IB) y GCP, y entre el IB y la recurrencia de la enfermedad. Método: Se analizaron los registros de 148 pacientes con melanoma maligno en estadios I y II sometidos a mapeo linfático y biopsia de GC desde 1999 hasta 2017 en una institución de tercer nivel de Córdoba, Argentina. Se realizaron linfocentellografía preoperatoria, mapeo linfático con técnica combinada y biopsia de GC. Se establecieron controles posoperatorios reglados a fin de detectar recurrencias. Resultados: Se identificó el GC en 145 pacientes (97.9\%) y resultó positivo en 22 (17.2\%). Se detectó recurrencia en 10 pacientes (8.3\%) con GC negativo (GCN), y en 2 (9.09\%) con GCP ( $p=0.188)$. La mediana del IB fue de $2 \mathrm{~mm}$ en los pacientes con GCP $y$ de $1.2 \mathrm{~mm}$ en los pacientes con GCN ( $p=0.002)$. La media del IB en los pacientes con recurrencia fue de $2.77 \mathrm{~mm}, y$ en los que no mostraron recaída fue de $2.01 \mathrm{~mm}(p=0.311)$. Conclusiones: La técnica combinada permite una alta tasa de identificación del GC. Se observó una mayor tendencia a la recurrencia en presencia de GCP. Se comprobó una relación estadísticamente significativa entre GCP e IB. La técnica del GC es efectiva y replicable en nuestro medio.

PALABRAS CLAVE: Melanoma maligno. Ganglio centinela. Biopsia de ganglio centinela. Índice de Breslow.

\begin{abstract}
Introduction: The study of the Sentinel Lymph Node (SLN) in Melanoma is a procedure that aims the identification of the first node to which the affected cutaneous sector drains in order to avoid unnecessary lymphadenectomies. The present study documents the frequency of identification of SLN; the relationship between positive SLN (PSLN) and recurrence, between the Breslow index (BI) and PSLN, and between BI and disease recurrence. Method: We analyzed the records of 148 patients with melanoma stages I and II undergoing lymphatic mapping and GC biopsy from 1999 to 2017 in a third level institution in Córdoba, Argentina. We performed preoperative lympho centellography, lymphatic mapping with combined technique and SLN biopsy. Postoperative controls were established in order to detect recurrences. Results: SLN was identified in 145 patients (97.9\%), being positive in 25 cases (17.2\%). Recurrence was detected in 10 (8.3\%) patients with negative SLN (NSLN), and in 2 (9.09\%) with PSLN ( $p=$ 0.188). The median BI was $2 \mathrm{~mm}$ in PCG patients and $1.2 \mathrm{~mm}$ in GCN patients $(p=0.002)$. The mean $B I$ in patients with recurrence was $2.77 \mathrm{~mm}$, and $2.01 \mathrm{~mm}$ in those who did not show relapse $(p=0.311)$. Conclusions: The combined technique allows a high GC identification rate. A greater tendency to recurrence was observed in the presence of CPG. A statistically significant relationship between GCP and IB was found. The GC technique is effective and replicable in our environment.
\end{abstract}

KEY WORDS: Melanoma. Sentinel lymph node. Sentinel lymph node biopsy. Breslow index.

Correspondencia:

*Manuel I. España

Naciones Unidas 346, CP5000, $\quad$ Fecha de recepción: 06-05-2018

Córdoba, Argentina Fecha de aceptación: 10-11-2018

E-mail: maesis17@gmail.com DOI: $10.24875 / C I R U .18000439$

Cir Cir. 2019;87:241-246

Contents available at PubMed www.cirugiaycirujanos.com 


\section{Introducción}

El melanoma maligno (MM) es una de las neoplasias que ha sufrido un mayor incremento en su incidencia en todo el mundo. Según la base de datos del Surveillance, Epidemiology, and End Results of $\mathrm{Na}$ tional Cancer Institute, se ha observado un aumento del $619 \%$ en el diagnóstico anual de MM entre los años 1950 y 2000, con 55,000 nuevos diagnósticos en 20041. En nuestro país existen escasos datos acerca de la incidencia de esta neoplasia.

La sobrevida a 5 años de los pacientes con MM localizado es de aproximadamente un $80 \%$, un $35 \%$ cuando hay metástasis ganglionar ${ }^{2}$ y un $10 \%$ cuando existen metástasis a distancia ${ }^{3,4}$, por lo que el diagnóstico y el tratamiento precoces son determinantes para la sobrevida de los pacientes ${ }^{5}$.

La primera mención del ganglio centinela (GC) la realizaron Gould, et al. ${ }^{6}$ en 1960 en pacientes con cáncer parotídeo, y luego $\mathrm{Cabanas}^{7}$ aplicó el concepto en el cáncer de pene.

En la década de 1990, Morton ${ }^{8}$ describió la técnica de biopsia del GC en el $\mathrm{MM}^{9,10}$, que se basa en el concepto de que las metástasis siguen una progresión ordenada a través de los canales linfáticos hasta la primera estación ganglionar antes de repartirse hacia las siguientes ${ }^{11}$. De esta manera, la técnica permitiría evitar linfadenectomías innecesarias.

Es por ello que el estudio del GC predice, con una precisión muy elevada, el estado del resto de los ganglios, evitando así la morbilidad que puede ocasionar la linfadenectomía convencional al reservar esta para aquellos casos en que el GC esté histopatológicamente afectado ${ }^{12,13}$. Se han utilizado distintos procedimientos para identificar el GC: Krag, en 1993, adaptó una sonda de detección de rayos gamma tras la inyección peritumoral de $99 \mathrm{mTc}$-sulfuro coloidal14,15 , y Albertini ${ }^{13,16}$ combinó dicho método con el azul vital utilizado por Morton ${ }^{9}$, técnica que empleamos actualmente.

El presente trabajo tiene por objetivo determinar la frecuencia de identificación y positividad del GC en nuestro medio, evaluar la relación entre la recurrencia del MM y la positividad del GC, y la relación del índice de Breslow con la positividad del GC y la recurrencia de la enfermedad.

\section{Método}

Se realizó un análisis retrospectivo de una base de datos prospectiva de registros clínicos de 148 pacientes sometidos a mapeo linfático de GC en el periodo comprendido entre septiembre de 1999 y abril de 2017.

Respecto a los criterios de inclusión, se utilizaron los de la séptima edición del American Joint Committee on Cancer. Se incluyeron pacientes de ambos sexos con diagnóstico de MM de miembros y tronco con índice de Breslow mayor de $1 \mathrm{~mm}$, índice de Breslow menor o igual a $1 \mathrm{~mm}$ con ulceración o más de una mitosis por campo, estadificación negativa y sometidos a mapeo linfático intraoperatorio y biopsia de GC.

Se consideraron criterios de exclusión el presentar MM cutáneo sin indicación de biopsia de GC (estadio 0 -tumor in situ- y 1 -índice de Breslow menor o igual a $1 \mathrm{~mm}$ sin ulceración y mitosis menor de una por campo-), la presencia de adenopatías palpables, la evidencia de enfermedad a distancia y la imposibilidad para identificar el GC durante la cirugía. También fueron excluidos los pacientes con MM ubicados en la cabeza y el cuello.

Se realizó linfocentellografía preoperatoria con 450 $\mathrm{mCi}$ de albúmina coloidal marcada $c{ }^{99} \mathrm{Tc}$ inyectada por vía intradérmica perilesional el día de la cirugía (máximo $8 \mathrm{~h}$ previas).

Se optó por anestesia regional o general en cada caso según la localización. Se infiltró azul patente epidérmico en los cuatro puntos cardinales de la lesión para lograr una doble marcación. Antes de seccionar la piel, se comprobó con una sonda gamma el punto más caliente de captación. Se procedió a la disección, la identificación y la resección del GC. Se comprobaron el nivel de radiación del ganglio una vez extraído y la presencia de radiación residual en el lecho quirúrgico, evaluando a partir de esta la necesidad de una disección adicional en caso de haber más de un GC.

El estudio del GC se hizo mediante una modificación de la técnica de Starz ${ }^{11}$, que consiste en seccionar el ganglio en cortes de $1 \mathrm{~mm}$ de espesor en sentido longitudinal. De dichas secciones se incluyen los cortes más sospechosos, que se procesan con la técnica habitual, inclusión en parafina y coloración con hematoxilina-eosina de tres niveles, para observarlos al microscopio óptico en busca de metástasis. Se realizaron además otros dos niveles para inmunomarcación con S100 y Melan A (hay que señalar que al inicio de nuestra experiencia también se utilizaba HMB45).

Se consideró GC positivo (GCP) aquel que fuera positivo para metástasis de MM con la técnica de hematoxilina-eosina o inmunomarcación, y GC negativo 
(GCN) a todo aquel que fuera negativo en todas ellas, siendo falsos negativos aquellos pacientes con GCN en los cuales se presentan metástasis en la zona ganglionar sometida a mapeo ${ }^{3}$.

En los pacientes en los que se encontró GCP se realizó linfadenectomía regional. La carga tumoral se informó según el método de Carlson, et al. ${ }^{17}$, quienes la clasifican en macrometástasis (>2 mm), micrometástasis $(<2 \mathrm{~mm}$ ), grupo de células (10-30 células en el seno marginal o espacio interfolicular) y células aisladas (1-20 células aisladas en el seno marginal).

Los controles posoperatorios se establecieron a los 15 y 30 días, y posteriormente cada 3 meses durante el primer año, cada 6 meses durante el segundo y anualmente a partir del tercer año. Los pacientes fueron evaluados mediante exploración clínica, pruebas de laboratorio (lactato deshidrogenasa), radiografía de tórax y ecografía abdominal. En algunos casos, según el criterio del médico clínico, se solicitó tomografía computada u otro estudio que se creyera pertinente.

Se consideró recaída local la que se presentó en el sitio del melanoma primario, y recaída regional la que se presentó en la zona ganglionar. Se tomó como asociada a falla en la técnica la presencia de recaída regional. No se consideró la aparición de metástasis a distancia sin recaída regional para valorar la técnica de GC.

Para el análisis estadístico, las variables continuas se expresaron como media y desviación estándar o como mediana y rangos intercuartílicos (RI) en función de su homogeneidad. Las variables categóricas se expresaron como número y porcentaje. Las variables categóricas se compararon con la prueba de ji al cuadrado o la prueba exacta de Fisher de acuerdo con las frecuencias esperadas. Las variables continuas se compararon con la prueba t de Student o la prueba de Mann-Whitney de acuerdo con su homogeneidad. Se consideró significativo un valor de $p<0.05$. El análisis se llevó a cabo mediante el software estadístico SPSS 22 (SPSS, Inc., Chicago, IL).

\section{Resultados}

De los 148 pacientes analizados, 81 fueron de sexo masculino (54\%) y la media de edad fue de 49 años (rango: 11-75) (Fig. 1). No se observaron diferencias significativas en la distribución de la edad según sexos.

La localización tumoral más frecuente fue en las extremidades, seguida del dorso, el pecho y

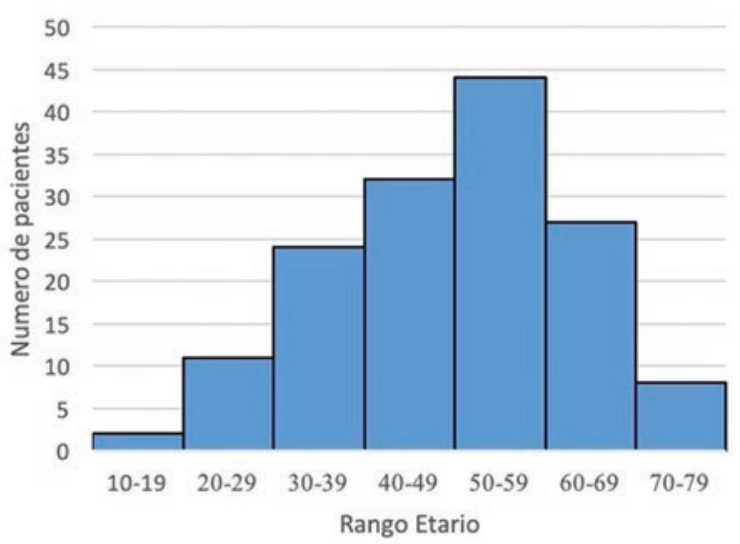

Figura 1. Distribución de los casos de melanoma maligno según rangos de edad $(n=148)$.

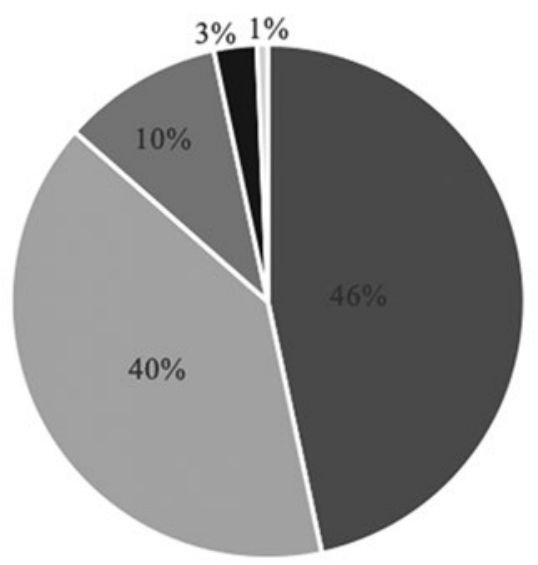

- Extremidades " Dorso " Pecho - Abdomen " otros.

Figura 2. Distribución de los casos de melanoma según la localización $(n=148)$.

el abdomen (Fig. 2). Un paciente presentó lesiones sincrónicas en el dorso y las extremidades $(0.7 \%)$, ambas con GCN y sin recidiva a la fecha (Fig. 2).

La mediana del índice de Breslow fue de $1.45 \mathrm{~mm}$ (RI: 1-2); la media del índice de Breslow en las mujeres fue de $2.18 \mathrm{~mm}$, mientras que en los hombres fue de $2.13 \mathrm{~mm}$ ( $p=$ no significativo).

Con respecto al GC, se logró identificarlo en 145 pacientes (97.9\%). En tres pacientes (2\%) no se logró la detección del GC por parte del cirujano durante el acto operatorio y se realizó linfadenectomía convencional. En 37 pacientes se identificaron dos GC, y en dos pacientes, tres GC (Tabla 1).

EI GC fue positivo en 25 casos (17.2\%) y negativo en 120 casos (82.7\%). En 22 de los 25 pacientes con GCP se realizó linfadenectomía programada. De los restantes casos, no se realizó la linfadenectomía en dos 


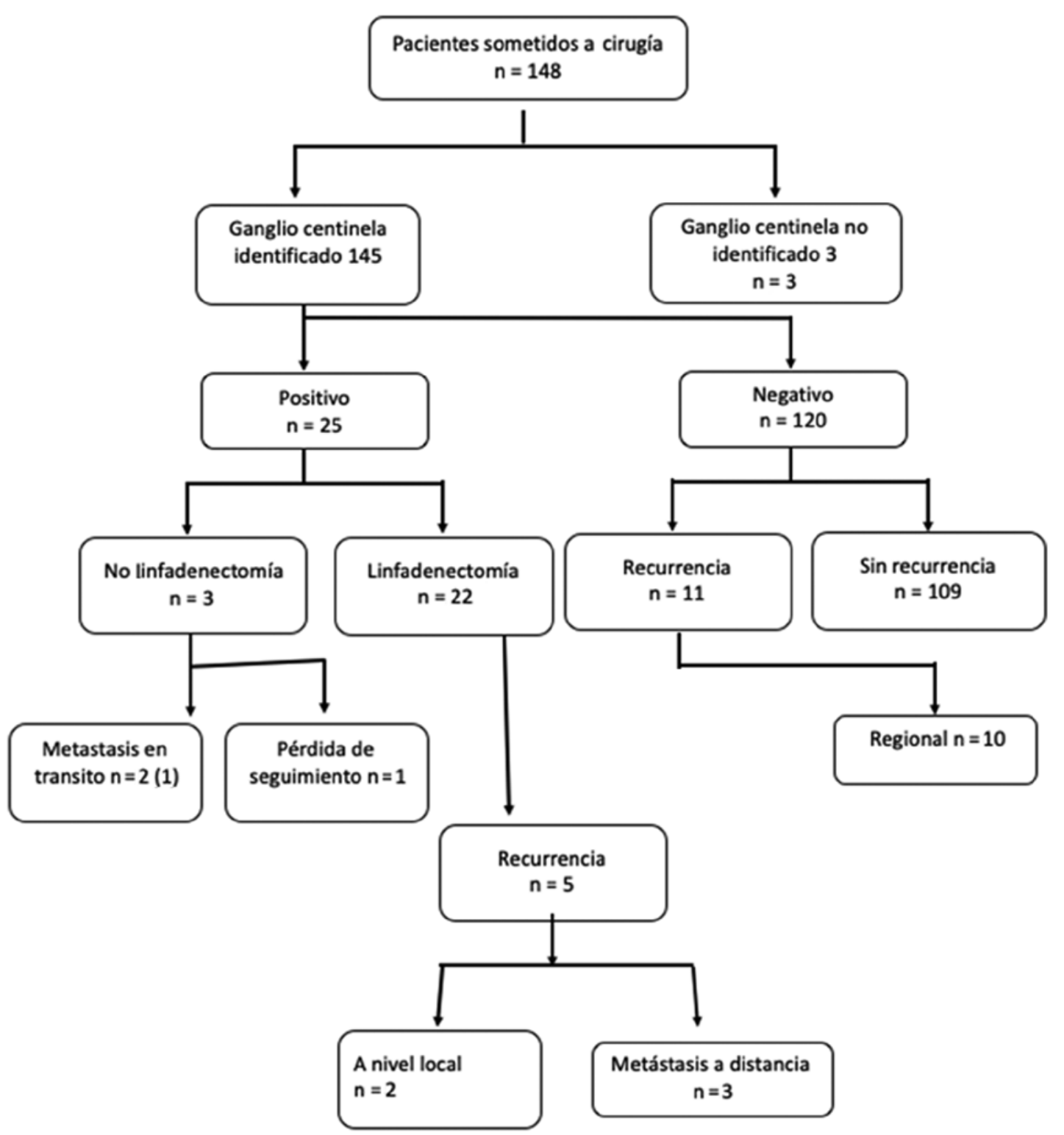

Figura 3. Conducta seguida con los pacientes sometidos al estudio. (1) No sometido a linfadenectomía por ser metástasis en tránsito.

Tabla 1. Número de ganglios identificados en la cirugía $(n=148)$

\begin{tabular}{lcc}
\hline Número de ganglios centinela & N & $\%$ \\
\hline 0 & 3 & 2,0 \\
1 & 106 & 71,6 \\
2 & 37 & 25,0 \\
3 & 2 & 1,4 \\
\hline
\end{tabular}

pacientes tras debatirlo en un comité multidisciplinario por tratarse de metástasis en tránsito con zona linfática negativa, y en el otro por abandono del seguimiento.

La media de seguimiento fue de 33.7 meses. La media de tiempo desde la cirugía hasta la detección de la recurrencia fue de 26.23 meses.
De los 120 pacientes con GCN, 10 (8.3\%) presentaron recaída regional a lo largo del seguimiento y uno tuvo recaída local. De los 22 pacientes con GCP sometidos a linfadenectomía, cinco presentaron recurrencia en el seguimiento. De estos, 2 (9.09\%) en los ganglios del sitio de la linfadenectomía, y el resto se manifestó con metástasis a distancia sin recaída regional. Al comparar la recaída regional entre GCN y GCP no se detectaron diferencias significativas (Fig. 3).

La mediana del índice de Breslow en los pacientes con GCP fue de $2 \mathrm{~mm}$ (RI: 1.5-3.3), mientras que en aquellos con GCN fue de $1.2 \mathrm{~mm}$ (RI: 0.8-2), siendo esta diferencia estadísticamente significativa ( $p=0.002)$. La media del índice de Breslow en los 
pacientes con recurrencia fue de $2.77 \mathrm{~mm}$, y de $2.01 \mathrm{~mm}$ para aquellos que no evidenciaron recaída a lo largo del seguimiento $(p=0.311)$.

\section{Discusión}

De acuerdo con lo ya establecido en estudios previos, el pronóstico para un paciente con diagnóstico de MM dependerá principalmente de la presencia de metástasis en los ganglios linfáticos regionales y del índice de Breslow ${ }^{18-21}$.

La biopsia del GC permite estudiar de forma selectiva los ganglios linfáticos de la cadena ganglionar regional con más riesgo de recibir las metástasis, limitando de esta manera la realización de tratamientos quirúrgicos más agresivos y con más comorbilidad, como la linfadenectomía ${ }^{22}$, que se reserva solo para aquellos pacientes con GCP. Así, el estado patológico del GC se convirtió en el factor pronóstico independiente más importante en términos de supervivencia global $^{18}$. La biopsia siempre debe ser analizada de manera diferida. Las secciones congeladas habituales no se recomiendan por el alto índice de falsos negativos ${ }^{11,19}$.

A partir de que en numerosos estudios retrospectivos se demostrara una fuerte correlación negativa entre la presencia de GCP y la supervivencia, la biopsia del GC se convirtió en una importante herramienta estadificadora y pronóstica 2,18,20.

También se ha documentado una relación directa entre el nivel de invasión vertical y la frecuencia de compromiso ganglionar regional, así como con la tasa de recurrencia, y esta relación es inversa con la sobrevida a 5 años ${ }^{21}$.

Con respecto al índice de Breslow, en nuestro estudio los GCP se relacionaron con MM primarios de mayor espesor. Al comparar con el grupo con GCN se observó una diferencia significativa. También se vio una tendencia de asociación entre el índice de Breslow en la lesión primaria y el riesgo de recurrencia de MM, aunque sin significancia estadística.

En nuestra serie, la tasa de identificación de GC mediante la técnica combinada de inyección perilesional con azul patente y linfocentellografía preoperatoria fue del $97.9 \%$, cifra similar a la reportada en la literatura ${ }^{13,16}$.

La principal limitación de la biopsia de GC son los falsos negativos, definidos como aquellos pacientes que presentan metástasis en los ganglios de un territorio ganglionar en el que, en el mismo momento, se les ha diagnosticado un $\mathrm{GCN}^{22}$. La tasa de falsos negativos se define como aquella fracción de pacientes con ganglios linfáticos comprometidos que no son detectados en el procedimiento y que luego se vuelven evidentes ${ }^{23}$. Las tasas de falsos negativos reportadas varían del 16 al $23 \%{ }^{24}$. Debido a que la recurrencia ganglionar puede tomar varios años hasta manifestarse clínicamente, a medida que aumenta el seguimiento evolutivo se incrementará la tasa de falsos negativos ${ }^{24}$. La recidiva regional ganglionar del MM tras la extirpación del GC con resultado anatomopatológico negativo varía, según los diferentes trabajos, del 0.8 al $4.1 \%^{11,25}$ tras un periodo de seguimiento de 2 años.

En cuanto a la recurrencia, en nuestra serie no pudo demostrarse una diferencia estadísticamente significativa entre los grupos con GCP y GCN, quizá debido al tamaño pequeño de la muestra, pero se observa una clara tendencia ${ }^{3,11,24}$.

El seguimiento medio en nuestra serie fue de 33.7 meses, cifra similar a la de otros estudios publicados $^{11,25}$ que se considera suficiente para valorar de forma efectiva la recurrencia posquirúrgica de MM y conocer la tasa de falsos negativos fidedigna.

El presente trabajo tiene como limitación el ser retrospectivo y su tamaño muestral; también su carácter unicéntrico restringe la extrapolación de resultados. Sin embargo, debido a los escasos datos propios de nuestra región con respecto a esta patología y la aplicación del mapeo de GC en MM, el presente estudio brinda información útil con respecto a la replicabilidad y los resultados de esta técnica en los países en desarrollo.

\section{Conclusión}

El grupo de pacientes con GCP se asoció significativamente a un Breslow mayor y se observó una tendencia a una mayor tasa de recurrencia a lo largo del seguimiento. La misma asociación se evidencia entre el espesor de Breslow y la recurrencia.

La técnica de GC es segura, efectiva y replicable en nuestro medio, con números similares a los reportados internacionalmente a pesar de las limitaciones propias de los países en desarrollo.

\section{Conflicto de intereses}

Los autores declaran no tener ningún conflicto de intereses. 


\section{Responsabilidades éticas}

Protección de personas y animales. Los autores declaran que para esta investigación no se han realizado experimentos en seres humanos ni en animales.

Confidencialidad de los datos. Los autores declaran que han seguido los protocolos de su centro de trabajo sobre la publicación de datos de pacientes.

Derecho a la privacidad y consentimiento informado. Los autores declaran que en este artículo no aparecen datos de pacientes.

\section{Bibliografía}

1. Jemal A, Tiwari RC, Murray $T$, Ghafoor A, Samuels A, Ward E, et al. Cancer statistics, 2004. CA Cancer J Clin. 2004;54:8-29.

2. Bleicher RJ, Essner R, Foshag LJ, Wanek LA, Morton DL. Role of sentinel lymphadenectomy in thin invasive cutaneous melanomas. J Clin Oncol. 2003;21:1326-31.

3. Li L-XL, Scolyer RA, Ka VSK, McKinnon JG, Shaw HM, McCarthy SW, et al. Pathologic review of negative sentinel lymph nodes in melanoma patients with regional recurrence: a clinicopathologic study of 1152 patients undergoing sentinel lymph node biopsy. Am J Surg Pathol. 2003;27:1197-202.

4. Mangas C, Paradelo C, Puig S, Gallardo F, Marcoval J, Azon A, et al. [Initial evaluation, diagnosis, staging, treatment, and follow-up of patients with primary cutaneous malignant melanoma. Consensus statement of the Network of Catalan and Balearic Melanoma Centers]. Actas Dermosifiliogr. 2010;101:129-42.

5. Garbe C, Eigentler TK. Diagnosis and treatment of cutaneous melanoma: state of the art 2006. Melanoma Res. 2007;17:117-27.

6. Gould EA, Winship T, Philbin PH, Kerr HH. Observations on a "sentinel node" in cancer of the parotid. Cancer. 1960;13:77-8.

7. Cabanas RM. An approach for the treatment of penile carcinoma. Cancer. 1977:39:456-66.

8. Morton DL. Current management of malignant melanoma. Ann Surg 1990;212:123-4.

9. Morton DL, Wen DR, Wong JH, Economou JS, Cagle LA, Storm FK et al. Technical details of intraoperative lymphatic mapping for early stage melanoma. Arch Surg. 1992;127:392-9.

10. Cochran AJ, Wen DR, Morton DL. Management of the regional lymph nodes in patients with cutaneous malignant melanoma. World J Surg. 1992;16:214-21.
11. Gershenwald JE, Colome MI, Lee JE, Mansfield PF, Tseng C, Lee JJ, et al. Patterns of recurrence following a negative sentinel lymph node biopsy in 243 patients with stage I or II melanoma. J Clin Oncol. 1998;16:2253-60.

12. Thompson JF, McCarthy WH, Bosch CM, O'Brien CJ, Quinn MJ Paramaesvaran S, et al. Sentinel lymph node status as an indicator of the presence of metastatic melanoma in regional lymph nodes. Melanoma Res. 1995;5:255-60.

13. Albertini JJ, Cruse CW, Rapaport D, Wells K, Ross M, DeConti R, et al. Intraoperative radio-lympho-scintigraphy improves sentinel lymph node identification for patients with melanoma. Ann Surg. 1996; 223:217-24

14. Krag DN, Meijer SJ, Weaver DL, Loggie BW, Harlow SP, Tanabe KK, et al. Minimal-access surgery for staging of malignant melanoma. Arch Surg. 1995;130:654-60.

15. Alex JC, Weaver DL, Fairbank JT, Rankin BS, Krag DN. Gamma-probe-guided lymph node localization in malignant melanoma. Surg Oncol. 1993;2:303-8.

16. Bostick P, Essner R, Glass E, Kelley M, Sarantou T, Foshag LJ, et al. Comparison of blue dye and probe-assisted intraoperative lymphatic mapping in melanoma to identify sentinel nodes in 100 lymphatic basins. Arch Surg. 1999;134:43-9.

17. Carlson GW, Murray DR, Lyles RH, Staley CA, Hestley A, Cohen C. The amount of metastatic melanoma in a sentinel lymph node: does it have prognostic significance? Ann Surg Oncol. 2003;10:575-81.

18. Morton DL, Thompson JF, Cochran AJ, Mozzillo N, Nieweg OE, Roses DF, et al. Final trial report of sentinel-node biopsy versus nodal observation in melanoma. N Engl J Med. 2014;370:599-609.

19. Yu LL, Flotte TJ, Tanabe KK, Gadd MA, Cosimi AB, Sober AJ, et al. Detection of microscopic melanoma metastases in sentinel lymph nodes. Cancer. 1999;86:617-27.

20. Milton GW, Shaw HM, McCarthy WH, Pearson L, Balch CM, Soong SJ. Prophylactic lymph node dissection in clinical stage I cutaneous malignant melanoma: results of surgical treatment in 1319 patients. Br J Surg. 1982;69:108-11.

21. Clary BM, Brady MS, Lewis JJ, Coit DG. Sentinel lymph node biopsy in the management of patients with primary cutaneous melanoma: review of a large single-institutional experience with an emphasis on recurrence. Ann Surg. 2001;233:250-8.

22. Shivers SC, Wang X, Li W, Joseph E, Messina J, Glass LF, et al. Molecular staging of malignant melanoma: correlation with clinical outcome. JAMA. 1998;280:1410-5

23. Tsao $H$, Atkins MB, Sober AJ. Management of cutaneous melanoma. N Engl J Med. 2004;351:998-1012.

24. Balch CM, Soong SJ, Murad TM, Ingalls AL, Maddox WA. A multifactorial analysis of melanoma. II. Prognostic factors in patients with stage I (localized) melanoma. Surgery. 1979;86:343-51.

25. Ramnath, Kamath, Brobeil, Stall A, Kamath V V, Cruse CW, et al. Lymphatic mapping for melanoma: long-term results of regional nodal sampling with radioguided surgery. Cancer Control. 1997;4:483-90. 